\title{
COMPLEX RETINAL DETACHMENT IN PHAKIC PATIENTS
}

\section{Previtrectomy Phacoemulsification Versus Combined Phacovitrectomy}

\author{
GIAN MARCO TOSI, MD, PhD,* ANGELO BALESTRAZZI, MD, PhD,* STEFANO BAIOCCHI, MD,* \\ ANTONIO TARANTELLO, MD,* GABRIELE CEVENINI, MD, $\dagger$ DAVIDE MARIGLIANI, MD,* \\ FRANCESCO SIMI, MD*
}

\begin{abstract}
Purpose: To assess the impact of phacoemulsification performed one week before pars plana vitrectomy versus combined phacovitrectomy on postoperative anterior segment status and final functional and anatomical outcomes in phakic patients affected by complex rhegmatogenous retinal detachment.

Methods: The authors retrospectively reviewed the records of 59 phakic patients affected by complex rhegmatogenous retinal detachment. Twenty-nine patients underwent cataract surgery 7 days before vitrectomy (preemptive cataract surgery-Group 1), whereas 30 patients underwent combined phacovitrectomy (Group 2). Preoperative, intraoperative, early- and late-postoperative outcomes were measured and compared.

Results: Numbers of previous retinal surgical procedures, nuclear sclerosis grade, proliferative vitreoretinopathy grade, eyes with inferior breaks, surgical time, and ratio of silicone oil/gas tamponade were all similar between the two groups. After surgery, there was less extension of posterior synechia in Group 1. There was no significant difference in fibrin, number of patients with posterior synechia, final intraocular pressure, retinal redetachment rate, final retinal status, or final best-corrected visual acuity.

Conclusion: Preemptive cataract surgery was associated with less extensive postoperative posterior synechia, however, its final functional and anatomical outcomes were not significantly different from those of phacovitrectomy. Both approaches were efficacious.

RETINA 37:630-636, 2017
\end{abstract}

$\mathrm{P}$ ars plana vitrectomy (PPV) is often necessary to repair complex rhegmatogenous retinal detachment (RRD),${ }^{1-4}$ and lens status may determine the anatomical success in such patients. ${ }^{3,5}$ A preexisting cataract may limit visualization, the native lens may limit the extent to which the vitreous can be

From the *Ophthalmology Unit, Department of Medicine, Surgery and Neuroscience, University of Siena, Siena, Italy; and $†$ Department of Medical Biotechnologies, University of Siena, Siena, Italy.

None of the authors have any financial/conflicting interests to disclose.

This is an open access article distributed under the terms of the Creative Commons Attribution-NonCommercial-NoDerivatives License 4.0 (CC BY-NC-ND), which permits downloading and sharing the work provided it is properly cited. The work cannot be changed in any way or used commercially.

Reprint requests: Gian Marco. Tosi, MD, PhD, Ophthalmology Unit, Department of Medicine, Surgery and Neuroscience, University of Siena, Siena University Hospital, Viale Bracci 1, 53100 Siena, Italy; e-mail: gmtosi@ tin.it removed and traction relieved, and peripheral pathology may be more difficult to identify and treat in the phakic eye. , $^{2,5-13}$

Eyes left phakic at the completion of surgery may have a lower rate of anatomical success when compared with eyes rendered aphakic or pseudophakic., ${ }^{2,33-16}$ Pars plana vitrectomy associated with silicone oil or gas tamponade in a phakic eye leads to cataract. Oil removal is usually performed before cataract surgery, meaning multiple surgeries are needed in the case of redetachment. Moreover, cataract surgery may be more complex in the previously vitrectomized eye. ${ }^{17-19}$

Management options to render the eye nonphakic during vitrectomy include phacofragmentation through the pars plana (most often without intraocular lens-IOL) and combination anterior cataract surgery (with IOL). There are advantages to each approach., $3,6,10-12,20-26$ 
An alternative is to render the eye nonphakic before PPV (preemptive cataract surgery with IOL placement). The outcomes of this last approach have not been reported to date. We compared two groups of patients with phakic complex RRD from the specific view points of their postoperative anterior segment status and final anatomical and functional outcomes. In the first Group, phacoemulsification with IOL was performed one week before PPV (preemptive cataract surgery), whereas in the second Group, phacoemulsification with IOL was performed with PPV in the same sitting (combined phacovitrectomy).

\section{Patients and Methods}

We retrospectively reviewed the clinical records of patients affected by RRD who were subjected to PPV between January 2011 and March 2015 at the Ophthalmology Unit of the Department of Medicine, Surgery and Neuroscience, University of Siena, Siena, Italy. The research adhered to the principles of the Declaration of Helsinki, and the institutional review board approved the study. Patients were treated after being informed of the nature of the treatment being offered, the potential risks, benefits, adverse effects, possible treatment outcomes, and after having signed a consent form.

The patients included had clinical signs of primary or recurrent chronic total or subtotal macula-involving complex RRD (RRD extent $270^{\circ}$ or greater, very low mobility, rolled edges of retinal breaks, or wellestablished proliferative vitreoretinopathy (PVR) with epiretinal and/or subretinal proliferation) and had not previously been subjected to cataract surgery.

We excluded patients with recent onset partial, subtotal, or total phakic RRD, recent and late onset pseudophakic or aphakic RRD, tractional diabetic retinal detachment, RRD due to giant retinal tear, RRD related to ocular traumas, and patients subjected to previous intraocular surgery except vitreoretinal surgery.

Cataract surgery was performed either 1 week (range: $6-8$ days; mean: 7 days) before PPV (preemptive cataract surgery-Group 1) or during the PPV procedure (combined phacovitrectomy-Group 2). In particular, in Group 1 cataract surgery was performed at the time of the initial visit and PPV 6 days to 8 days (mean: 7 days) after cataract surgery. In the combined Group, the surgical procedure was performed between 3 and 6 days after initial diagnosis.

The patients were assigned to either Group 1 or Group 2 based on the logistics and timing of repair, that is, patient preference, available operating room time, etc.

Phacoemulsification was performed by one of three surgeons (A.B., S.B. or G.M.T.) in both Groups.
Vitrectomy was performed by the same surgeon (G. M.T.) assisted by a vitreoretinal fellow.

The cataract procedure, the IOL inserted (aspheric, hydrophobic acrylic, foldable, one-piece IOL [AcrySof SA60AT; Alcon Inc, Fort Worth, TX]), and the phacoemulsification machine (Infiniti Vision System; Alcon Inc), were identical in Group 1 and Group 2. The cataract wound was sutured in every patient in both Groups. No intracameral steroids were used at the conclusion of the cataract surgery in either Group. Intraocular lens calculation was based on the biometric findings and anteroposterior globe measurements of both eyes, as well as the opposite eye refraction.

In patients who underwent preemptive cataract surgery, a posterior capsulectomy was not performed at the time of PPV.

Encircling scleral buckling was associated with PPV in all patients using a 41 band. For the patients who had previously been subjected to scleral buckling for RRD, no scleral buckling revision was performed. In both Groups, extensive 3-port 20- or 23-gauge PPV was performed using the Stellaris PC (Bausch and Lomb, Rochester, NY) and a panoramic contact viewing system (Advanced Visual Instruments, Inc, New York, NY). Twenty-gauge PPV was chosen because of the temporary absence of appropriate instrumentation for bimanual membrane dissection in small-gauge PPV. Epiretinal membrane peeling and retinotomy were performed when necessary and appropriate. Intraocular tamponade was achieved with either silicone oil $1000 \mathrm{cSt}$ or gas (C3F8 13\%). Silicone oil was used if the patient was monocular; when maintaining prone positioning was too difficult; when a retinotomy of more than $90^{\circ}$ was required; and at the discretion of the operating surgeon.

Postoperative management after preemptive cataract surgery consisted of the topical instillation of tobramycindexamethasone four times daily, moxifloxacin three times daily, and bromfenac twice daily, up to the day of the PPV procedure.

Postoperative management after PPV for both Groups consisted of the topical instillation of tobramycindexamethasone four times daily, bromfenac twice daily, and cyclopentolate twice daily.

Preoperative data were reviewed together with postoperative data from days $1,7,15$, and 22 , and months 1, 2, 3, and 6. For eyes with longer follow-up periods, subsequent information was also reviewed.

The following preoperative variables were identified: patient age/sex, number and type of previous retinal surgeries, best-corrected visual acuity, anterior segment status, intraocular pressure (IOP), and RRD characteristics. In particular, we recorded the corneal endothelial cell count, degree of nuclear sclerosis (graded from 1 to 
4), location of retinal breaks, and extent of PVR (updated PVR classification). ${ }^{27}$ For the intergroup comparison of preoperative retinal status, we divided the PVR grade $\mathrm{C}$ into two subgroups, the first lower than C6 and the second equal to or greater than C6.

The intraoperative information recorded included phacoemulsification- or PPV-associated complications, PPV surgical time, type of PPV (noncannulated 20 gauge or protected 23 gauge), PPV-associated procedures (scleral buckle and PVR removal), and postoperative tamponade.

Postoperative outcomes were divided into early postoperative (first 6 months after surgery) and late postoperative (more than 6 months after surgery). The early postoperative outcomes were presence of corneal edema, first postoperative day IOP, use of IOPlowering medication in the first 3 weeks after PPV, formation of fibrin and posterior synechia, extent of posterior synechia, occurrence of IOL dislocation, occurrence of pupillary block, occurrence of optic/iris capture, and whether postoperative prone positioning was recommended. In the early postoperative period, IOP-lowering medications were initiated if the IOP was above $25 \mathrm{mmHg}$. Each topical antiglaucoma medication was given a score of 1 , and an additional 1 point was calculated if a systemic anhydrase inhibitor was added. Fibrin in the anterior chamber was documented by its presence or absence, without grading its severity. The extent of posterior synechia was noted and documented after dilatation of the pupil with tropicamide, according to the extent of pupillary margin involvement (less than $90^{\circ}$ [ 1 point], between $90^{\circ}$ and $180^{\circ}$ [2 points], between $180^{\circ}$ and $270^{\circ}$ [3 points], and more than $270^{\circ}$ [ 4 points]).

The late postoperative outcomes included final IOP including the number of patients with hypotony (IOP $\leq 5 \mathrm{mmHg}$ ); patients on IOP-lowering medications to maintain an IOP $\leq 20 \mathrm{mmHg}$; number of patients with posterior synechia and extent of posterior synechia; number of patients with optic iris capture; cystoid macular edema; occurrence of postoperative macular epiretinal membrane; recurrence of retinal detachment and its cause; number of patients with oil in the eye at last follow-up; final best-corrected visual acuity; and final refractive status.

\section{Statistical Analysis}

Group comparisons were performed using the Student's $t$-test for quantitative continuous variables; the nonparametric test of medians for quantitative discrete variables; and the Fisher exact test (applied to contingency tables) for qualitative variables. A Bonferroni correction of the $P$ value was not used because only one comparison (between the two Groups) was performed for each variable. A statistical significance level of $95 \%$ was used for all computations $(P=0.05)$.

\section{Results}

\section{Demographics}

After the application of inclusion and exclusion criteria, 73 patients were identified. Fourteen patients were unable to attend the follow-up visit and were not included in the study. A total of 59 eyes of 59 subjects participated in the study. The mean age was $64.8 \pm$ 10.4 years; 37 patients were male and 22 were female.

Twenty-nine eyes constituted Group 1 (preemptive cataract surgery) and 30 eyes constituted Group 2 (combined phacovitrectomy Group). Follow-up ranged from 9 months to 48 months in Group 1 (mean 21.1 months) and from 9 to 48 months in Group 2 (mean 23.6 months) $(P=0.412)$.

\section{Entry and Intraoperative Findings}

At entry, a significantly worse best-corrected visual acuity was found in Group 1 compared with Group 2 $(P<0.001)$ (Table 1$)$. One patient had a posterior capsular break in the preemptive cataract surgery Group (3.4\%), whereas no patient had a posterior capsular break in the combined Group. In the patient with capsular break, the IOL was implanted in the capsular bag and the patient underwent PPV 7 days later, receiving $\mathrm{C} 3 \mathrm{~F} 813 \%$ as a postoperative tamponade, without developing postoperative posterior synechia or IOL dislocation. No patient required the use of iris retractors in Group 1, whereas 2 patients required the use of iris retractors in Group 2 because of sudden intraoperative myosis during the phacoemulsification procedure $(P=0.364)$. No other complication was encountered during phacoemulsification and the PPV procedure in either Group. In Group 1, five patients required retinotomy (17.2\%), whereas in Group 2, four patients required retinotomy $(13.3 \%)(P=0.731)$.

\section{Early Postoperative Findings}

No patients in either Group experienced corneal edema on postoperative day 1 (Table 2). The first postoperative day IOP was significantly lower in Group 1 $(25.66 \pm 5.7 \mathrm{mmHg})$ compared with Group $2(29.77 \pm$ $7.6 \mathrm{mmHg})(P=0.022)$, as was the mean number of IOP-lowering medications at Week $1(P=0.005), 2$ $(P=0.003)$, and $3(P=0.035)$ after surgery. Fibrin formation was lower in Group 1, although not significantly, ( 1 of 29 eyes [3.4\%]) compared with Group 2 
Table 1. Entry and Intraoperative Variables of Group 1 Patients (Preemptive Cataract Surgery) and of Group 2 Patients (Combined Phacovitrectomy)

\begin{tabular}{|c|c|c|c|}
\hline & Group 1 (29 Patients) & Group 2 (30 Patients) & $P$ \\
\hline Sex, n (\%) & & & 0.288 \\
\hline Male & $16(55.2)$ & $21(70)$ & \\
\hline Female & $13(44.8)$ & $9(30)$ & \\
\hline Mean age & $66.2 \pm 9.9$ & $63.9 \pm 10.3$ & 0.385 \\
\hline Previous surgery, $n(\%)$ & $4(13.8)$ & $7(23.3)$ & 0.506 \\
\hline Entrance VA, n (\%) & & & 0.001 \\
\hline CF & $2(6.9)$ & $21(70)$ & \\
\hline $\mathrm{HM}$ & $22(75.8)$ & $7(23.3)$ & \\
\hline LP & $5(17.2)$ & $2(6.6)$ & \\
\hline Mean preop IOP, mmHg & $15 \pm 3$ & $13.8 \pm 4.7$ & 0.244 \\
\hline Nuclear sclerosis, n (\%) & & & 0.989 \\
\hline Grade 1 & $12(41.3)$ & $13(43.3)$ & \\
\hline Grade 2 & $13(44.8)$ & $13(43.3)$ & \\
\hline Grade 3 & 4 (13.8) & $4(13.3)$ & \\
\hline Inferior breaks & $14(48.2)$ & $15(50)$ & 1.000 \\
\hline PVR grade, n (\%) & & & 0.296 \\
\hline A & 6 (20.6) & $10(33.3)$ & \\
\hline B & 11 (37.9) & $12(40)$ & \\
\hline$<\mathrm{C} 6$ & $7(24.1)$ & $7(23.3)$ & \\
\hline$\geq \mathrm{C} 6$ & $5(17.2)$ & $1(3.3)$ & \\
\hline PPV caliper, n (\%) & & & 0.748 \\
\hline $20 \mathrm{G}$ & $5(17.2)$ & 7 (23.3) & \\
\hline $23 \mathrm{G}$ & $24(82.8)$ & $23(76.6)$ & \\
\hline $\begin{array}{l}\text { Need for iris retractors during PPV, } \mathrm{n} \\
(\%)\end{array}$ & $0(0)$ & $2(6.6)$ & 0.364 \\
\hline Retinotomy, n (\%) & $5(17.2)$ & $4(13.3)$ & 0.731 \\
\hline Tamponade, n (\%) & & & 1.000 \\
\hline Silicone oil & $14(48.2)$ & $15(50)$ & \\
\hline Gas & $15(51.7)$ & $15(50)$ & \\
\hline Mean surgical time, minutes & $178.5 \pm 34.4$ & $176.9 \pm 27.7$ & 0.816 \\
\hline Posterior capsule break, n (\%) & $1(3.4)$ & $0(0)$ & 0.246 \\
\hline
\end{tabular}

$\mathrm{CF}$, count fingers; HM, hand motion; LP, light perception; VA, visual acuity. Statistically significant values are in italics.

(6 of 30 eyes [20\%]) $(P=0.103)$. No significant between-group difference was found in terms of the number of patients with posterior synechia (3 of 29 in Group 1 [10.3\%] and 7 of 30 in Group 2 [23.3\%]) $(P=0.118)$, whereas the extension of posterior synechia was lower in Group $1(P=0.019)$. In fact, a total of 4 quadrants of pupillary margin were involved in Group 1 , whereas a total of 14 quadrants were involved in Group 2. The 2 patients who needed iris retractors in Group 2 did not develop fibrin but did develop posterior synechia. Among the 6 patients in Group 2 who showed fibrin, 4 developed posterior synechia; the 1 patient in Group 1 showing fibrin did not develop posterior synechia. No patients in either Group experienced IOL dislocation, optic/iris capture, or pupillary block.

\section{Late Postoperative Findings}

No significant differences were found between Groups 1 and 2 in terms of final IOP $(P=0.560)$ (Table $3)$. With reference to the 3 hypotony patients ( 1 in
Group 1 and 2 in Group 2), the 2 patients in Group 2 showed fibrin formation and developed 3 and 4 quadrants of posterior synechia, respectively. Neither of these two showed recurrent RRD. The patient in Group 1 did not develop fibrin nor posterior synechia but suffered recurrent retinal detachment from PVR.

All the recurrences of RRD ( 1 in Group 1 and 3 in Group 2) were related to PVR; none of the patients with recurrent RRD showed postoperative fibrin or posterior synechia.

\section{Discussion}

In complex retinal detachment, eyes managed with PPV and left phakic at the completion of surgery may have a lower rate of anatomical success compared with eyes rendered aphakic or pseudophakic., ${ }^{3,14-16}$ Even in uncomplicated RRD, although the results are not always conclusive, ${ }^{4,28}$ Orlin et $\mathrm{al}^{13}$ and Caiado et $\mathrm{al}^{2}$ found a significantly increased redetachment rate in 
Table 2. Early Postoperative Variables of Group 1 Patients (Preemptive Cataract Surgery) and of Group 2 Patients (Combined Phacovitrectomy)

\begin{tabular}{|c|c|c|c|}
\hline & Group 1 (29 Patients) & Group 2 (30 Patients) & $P$ \\
\hline Corneal edema & 0 & 0 & 1.000 \\
\hline Mean postop IOP (Day 1), mmHg & $25.6 \pm 5.7$ & $29.7 \pm 7.6$ & 0.022 \\
\hline $\begin{array}{l}\text { Mean no. postop antiglaucoma } \\
\text { medications (Week 1) }\end{array}$ & $0.62 \pm 0.7$ & $1.23 \pm 0.9$ & 0.005 \\
\hline $\begin{array}{l}\text { Mean no. postop antiglaucoma } \\
\text { medications (Week 2) }\end{array}$ & $0.37 \pm 0.7$ & $1.16 \pm 1.2$ & 0.003 \\
\hline $\begin{array}{l}\text { Mean no. postop antiglaucoma } \\
\text { medications (Week 3) }\end{array}$ & $0.24 \pm 0.5$ & $0.76 \pm 1.2$ & 0.035 \\
\hline Prone position, $\mathrm{n}(\%)$ & $14(48.2)$ & $15(50)$ & 1.000 \\
\hline Fibrin, n (\%) & $1(3.4)$ & $6(20)$ & 0.103 \\
\hline Posterior synechia, n (\%) & $3(10.3)$ & 7 (23.3) & 0.118 \\
\hline \multicolumn{4}{|l|}{$\begin{array}{l}\text { Synechia extension among patients } \\
\text { with posterior synechia, } \mathrm{n}(\%)\end{array}$} \\
\hline $0^{\circ}-90^{\circ}$ & $2(66.6)$ & $3(42.8)$ & \\
\hline $90^{\circ}-180^{\circ}$ & 1 (33.3) & $2(28.5)$ & \\
\hline $180^{\circ}-270^{\circ}$ & $0(0)$ & 1 (14.2) & \\
\hline $270^{\circ}-360^{\circ}$ & $0(0)$ & 1 (14.2) & \\
\hline IOL dislocation & 0 & 0 & 1.000 \\
\hline Pupillary block & 0 & 0 & 1.000 \\
\hline Optic/iris capture & 0 & 0 & 1.000 \\
\hline
\end{tabular}

Statistically significant values are in italics.

phakic compared with pseudophakic/aphakic eyes. In addition, Caiado et $\mathrm{al}^{2}$ demonstrated the importance of combined phacovitrectomy in relation to improved outcomes in RRD repair, with success rates similar to primary PPV in pseudophakic eyes. Both Orlin et $\mathrm{al}^{13}$ and Caiado et $\mathrm{al}^{2}$ hypothesized more extensive vitreous removal to be the major reason for increased reattachment rates in pseudophakic or aphakic PPV compared with phakic PPV. They included patients without $\mathrm{PVR}^{13}$ or with a PVR grade of up to $\mathrm{CP} 1 .^{2}$

Management options to render the eye nonphakic during vitrectomy include combination anterior

Table 3. Late Postoperative Variables of Group 1 Patients (Preemptive Cataract Surgery) and of Group 2 Patients (Combined Phacovitrectomy)

\begin{tabular}{|c|c|c|c|}
\hline & Group 1 (29 Patients) & Group 2 (30 Patients) & $P$ \\
\hline Final IOP, n (\%) & & & 0.560 \\
\hline Norm & $26(89.6)$ & $24(80)$ & \\
\hline Нyро & $1(3.4)$ & $2(6.6)$ & \\
\hline Hyper & $2(6.8)$ & 4 (13.3) & \\
\hline Posterior synechia & 3 (10.3) & 7 (23.3) & 0.118 \\
\hline $\begin{array}{l}\text { Synechia extension among patients } \\
\text { with posterior synechia, } n(\%)\end{array}$ & & & 0.019 \\
\hline $0^{\circ}-90^{\circ}$ & $2(66.6)$ & $3(42.8)$ & \\
\hline $90^{\circ}-180^{\circ}$ & $1(33.3)$ & $2(28.5)$ & \\
\hline $180^{\circ}-270^{\circ}$ & $0(0)$ & $1(14.2)$ & \\
\hline $270^{\circ}-360^{\circ}$ & $0(0)$ & $1(14.2)$ & \\
\hline Optic/iris capture & 0 & 0 & 1.000 \\
\hline CME, n (\%) & $0(0)$ & $0(0)$ & 1.000 \\
\hline ERM, n (\%) & $7(24.1)$ & $4(13.3)$ & 0.333 \\
\hline Recurrent RRD, n (\%) & $1(3.4)$ & $3(10)$ & 0.483 \\
\hline Recurrence due to PVR, n (\%) & $1(100)$ & $3(100)$ & 0.483 \\
\hline Final retinal reattachment, $\mathrm{n}(\%)$ & $29(100)$ & $30(100)$ & 1.000 \\
\hline $\begin{array}{l}\text { Eyes with permanent silicone oil, } n \\
(\%)\end{array}$ & $1(3.4)$ & $1(3.3)$ & 0.746 \\
\hline Mean final BCVA, logMAR & $0.42 \pm 0.65$ & $0.45 \pm 0.63$ & 0.859 \\
\hline Mean refractive status & $-1.86 \pm 0.75$ & $-1.99 \pm 1.59$ & 0.687 \\
\hline
\end{tabular}

BCVA, best-corrected visual acuity; CME, cystoid macular edema; ERM, epiretinal membrane.

Statistically significant values are in italics. 
cataract surgery and phacofragmentation through the pars plana.

Phacofragmentation, by removing the potential scaffold for reproliferation constituted by the lens and the capsule, is reserved primarily for advanced PVR with a significant anterior component. ${ }^{3,5}$

Combination cataract surgery (with IOL) with vitrectomy (combined phacovitrectomy) affords enhanced access to and management of the anterior pathology when compared with phakic vitrectomy. However, combined phacovitrectomy may lead to anterior segment complications such as optic/iris capture, posterior synechia, capsular phimosis, and secondary pupillary block. ${ }^{3,5,6,10,12,20,21}$ This development may be due to the displacement of the lens anteriorly from the vitreous tamponade, possibly in combination with an inflammatory response after vitrectomy and endophotocoagulation. ${ }^{3,5}$ After combined phacovitrectomy, the anterior segment outcomes reported in the literature are variable, including up to $55.8 \%$ of patients with elevated IOP, ${ }^{6,14,18,21}$ between $4 \%$ and $22 \%$ with fibrin formation, ${ }^{6,18}$ between $7 \%$ and $30 \%$ with posterior synechia, ${ }^{11,18}$ and up to $33 \%$ with posterior capsule opacification. ${ }^{6,10,20,26}$ In addition, in case the vitreoretinal surgeon does not perform the cataract surgery, combined phacovitrectomy requires two surgeons, thus becoming logistically challenging.

An alternative is to render the eye nonphakic before PPV. Preemptive cataract surgery performed before planned vitreous surgery, although submitting the patient to two separate surgeries in a short period, may diminish the risk of these anterior segment/IOL complications, whereas maintaining the advantages of a pseudophakic vitrectomy. Rendering the eye pseudophakic 1 week before surgery may limit the degree of postvitrectomy inflammation and may allow the IOL to "sit firmly" in the capsule, thus limiting the risk of optic prolapse and capture.

To this end, we compared patients treated with phacoemulsification before PPV to patients who underwent combined phacovitrectomy. The 7-day time lapse between cataract surgery and PPV aimed to reduce anterior segment reaction without significantly postponing RRD surgery, possibly limiting further retinal damage.

We would have expected a longer surgical time in the combined group, however, no significant difference was encountered. This might be explained by the fact that cataract surgery is relatively brief, in an otherwise long surgical procedure because of the severity of preoperative retinal status, which in our custom is addressed with a meticulous vitreous removal and the placement of an encircling band.
The anatomical and functional outcomes after the surgical repair of these complex retinal detachments were similar between the Groups, perhaps because the retinal surgery was performed within the same relative period. All RRD recurrences were due to PVR; in particular, the recurrence rate was higher in the combined Group, although this was not statistically significant. In addition, the number of secondary macular epiretinal membranes was higher in the preemptive cataract Group; again, this was not significant. One patient in each Group required permanent silicone oil tamponade as a consequence of severe hypotony.

The anticipated purpose of preemptive cataract surgery was to reduce anterior segment inflammatory and mechanical complication rates after PPV with an extended vitreous tamponade. Fibrin formation and the number of patients with posterior synechia were higher in the combined Group, although not significantly. The extension of posterior synechia was significantly greater in the combined Group. This occurred in the absence of between-group differences in prone positioning. Meticulous vitreous removal was possible without the need for iris retractors in the preemptive cataract surgery Group, whereas two patients in the phacovitrectomy Group required iris retractors. These two patients developed posterior synechia. The absence of postoperative use of short acting mydriatics, which have been shown to reduce the incidence of posterior synechia, could have impacted our posterior synechia findings. ${ }^{11,12}$ However, the topically prescribed regimen was the same in both Groups. The slightly increased anterior segment inflammatory response in the combined Group may have been partially responsible for the early postoperative IOP findings. In fact, the phacovitrectomy Group showed a significantly higher first day postoperative IOP.

The fact that cell and flare were not evaluated and fibrin and posterior synechia were not quantitatively measured constitute limitations of this study. Moreover, the retrospective nature of the study and the lack of randomization in relation to the type of treatment constitute a bias. However, as previously noted, patient allocation to either preemptive or concurrent cataract surgery was based on logistical concerns (see Patients and Methods), and, in our opinion, had no effect on our results. In fact, because no significant between-group differences were encountered in sex, age, PVR grade, number of patients with inferior breaks, nuclear sclerosis, preoperative IOP, 20-gauge/23-gauge ratio, oil/gas ratio, surgical time, number of previous retinal surgeries, or number of patients who required prone positioning postoperatively, and because best-corrected visual acuity at entry, although significantly different 
between Groups, was no better than count fingers in either Group, the above-mentioned limitations may be considered of minor importance.

The peculiarities of this study are represented by the surgical approach/timing, which has never been reported so far, and by the select subcohort of patients affected by high-risk RRD. Preemptive cataract surgery in the management of phakic patients with complex RRD seems to at least slightly reduce the postoperative inflammatory component when compared with combined phacovitrectomy. However, in the present series, the two approaches had no significantly different final anatomical or functional outcomes. Both approaches were efficacious.

Key words: complex rhegmatogenous retinal detachment, pars plana vitrectomy, preemptive cataract surgery, combined phacovitrectomy, intraocular inflammation.

\section{References}

1. Adelman RA, Parnes AJ, Sipperley JO, Ducournau D; European Vitreo-Retinal Society (EVRS) Retinal Detachment Study Group. Strategy for the management of complex retinal detachments: the European vitreo-retinal society retinal detachment study report 2. Ophthalmology 2013;120:1809-1813.

2. Caiado RR, Magalhães O Jr., Badaró E, et al. Effect of lens status in the surgical success of 23-gauge primary vitrectomy for the management of rhegmatogenous retinal detachment: the Pan American Collaborative Retina Study (PACORES) group results. Retina 2015;35:326-333.

3. Tseng JJ, Schiff WM, Barile GR, et al. Influence of postoperative lens status on intraocular pressure in proliferative vitreoretinopathy. Am J Ophthalmol 2009;147:875-885.

4. Wong CW, Wong WL, Yeo IY, et al. Trends and factors related to outcomes for primary rhegmatogenous retinal detachment surgery in a large Asian tertiary eye center. Retina 2014;34:684-692.

5. Schiff WM, Barile GR, Hwang JC, et al. Diabetic vitrectomy: influence of lens status upon anatomic and visual outcomes. Ophthalmology 2007;114:544-550.

6. Smith M, Raman SV, Pappas G, et al. Phacovitrectomy for primary retinal detachment repair in presbyopes. Retina 2007;27:462-467.

7. Jain R, Newland H. Re: phacovitrectomy for primary retinal detachment repair in presbyopes. Retina 2008;28:666; author reply $666-667$.

8. Zhou P, Zhao MW, Li XX. Re: phacovitrectomy for primary retinal detachment repair in presbyopes. Retina 2008;28:665; author reply 665-666.

9. Er H. Comment on article by Smith M. Retina 2008;28:783; author reply 783-784.

10. Lee DY, Jeong HS, Sohn HJ, Nam DH. Combined 23-gauge sutureless vitrectomy and clear corneal phacoemulsification in patients with proliferative diabetic retinopathy. Retina 2011; 31:1753-1758.

11. Lee SB, Lee DG, Kwag JY, Kim JY. The effect of mydriatics on posterior synechia after combined pars plana vitrectomy, phacoemulsification, and intraocular lens implantation. Retina 2009;29:1150-1154.
12. Jun Hu Y, Chen WQ. Correspondence: the effects of two kinds of mydriatics on preventing postoperative posterior synechia. Retina 2010;30:186; author reply 186-187.

13. Orlin A, Hewing NJ, Nissen M, et al. Pars plana vitrectomy compared with pars plana vitrectomy combined with scleral buckle in the primary management of noncomplex rhegmatogenous retinal detachment. Retina 2014;34:1069-1075.

14. Storey P, Alshareef R, Khuthaila M, et al; Wills PVR Study Group. Pars plana vitrectomy and scleral buckle versus pars plana vitrectomy alone for patients with rhegmatogenous retinal detachment at high risk for proliferative vitreoretinopathy. Retina 2014;34:1945-1951.

15. Heimann H, Bartz-Schmidt KU, Bornfeld N, et al; Scleral Buckling versus Primary Vitrectomy in Rhegmatogenous Retinal Detachment Study Group. Scleral buckling versus primary vitrectomy in rhegmatogenous retinal detachment: a prospective randomized multicenter clinical study. Ophthalmology 2007;114:2142-2154.

16. Mendrinos E, Dang-Burgener NP, Stangos AN, et al. Primary vitrectomy without scleral buckling for pseudophakic rhegmatogenous retinal detachment. Am J Ophthalmol 2008;145:1063-1070.

17. Cole CJ, Charteris DG. Cataract extraction after retinal detachment repair by vitrectomy: visual outcome and complications. Eye (Lond) 2009;23:1377-1381.

18. Treumer F, Bunse A, Rudolf M, Roider J. Pars planavitrectomy, phacoemulsification and intraocular lens implantation. Comparison of clinical complications in a combined versus two-step surgical approach. Graefes Arch Clin Exp Ophthalmol 2006;244:808-815.

19. Chang MA, Parides MK, Chang S, Braunstein RE. Outcome of phacoemulsification after pars planavitrectomy. Ophthalmology 2002;109:948-954.

20. Chang CJ, Chang YH, Chiang SY, Lin LT. Comparison of clear corneal phacoemulsification combined with 25 -gauge transconjunctival sutureless vitrectomy and standard 20gauge vitrectomy for patients with cataract and vitreoretinal diseases. J Cataract Refract Surg 2005;31:1198-1207.

21. Pinarci EY, Bayar SA, Sizmaz S, et al. Anterior segment complications after phacovitrectomy in diabetic and nondiabetic patients. Eur J Ophthalmol 2013;23:223-229.

22. Demetriades AM, Gottsch JD, Thomsen R, et al. Combined phacoemulsification, intraocular lens implantation, and vitrectomy for eyes with coexisting cataract and vitreoretinal pathology. Am J Ophthalmol 2003;135:291-296.

23. Scharwey K, Pavlovic S, Jacobi KW. Combined clear corneal phacoemulsification, vitreoretinal surgery, and intraocular lens implantation. J Cataract Refract Surg 1999;25:693-698.

24. Goezinne F, Nuijts RM, Liem AT, et al. Corneal endothelial cell density after vitrectomy with silicone oil for complex retinal detachments. Retina 2014;34:228-236.

25. Friberg TR, Doran DL, Lazenby FL. The effect of vitreous and retinal surgery on corneal endothelial cell density. Ophthalmology 1984;91:1166-1169.

26. Rahman R, Briffa BV, Gupta A, Chinn DJ. Factors contributing to posterior capsule opacification following 23-gauge transconjunctival phacovitrectomy. Ophthalmic Surg Lasers Imaging 2011;42:229-233.

27. Machemer R, Aaberg TM, Freeman HM, et al. An updated classification of retinal detachment with proliferative vitreoretinopathy. Am J Ophthalmol 1991;112:159-165.

28. Kinori M, Moisseiev E, Shoshany N, et al. Comparison of pars plana vitrectomy with and without scleral buckle for the repair of primary rhegmatogenous retinal detachment. Am J Ophthalmol 2011;152:291-297. 